\title{
EBG ENHANCED FEEDS FOR HIGH APERTURE EFFICIENCY REFLECTOR ANTENNAS
}

\author{
Andrea Neto ${ }^{1}$, Nuria Llombart ${ }^{1}$, Giampiero Gerini ${ }^{1}$, Magnus Bonnedal ${ }^{2}$, and Peter De Maagt ${ }^{3}$ \\ ${ }^{1}$ TNO Defence, Safety and Security, 2597 AK Den Haag, The Netherlands. E-mail: andrea.neto, nuria.llombartjuan, \\ giampiero.gerini@tno.nl \\ ${ }^{2}$ Saab Ericsson Space, SE-405 15 Goteborg, Sweden.E-mail: magnus.bonnedal@space.se \\ ${ }^{3}$ ESA-ESTEC, 2200 AG Noordwijk, The Netherlands. E-mail: Peter.de.Maagt@esa.int
}

\section{INTRODUCTION}

Reflector antenna systems have been thoroughly investigated for decades and represent a mature technology, [1]. Major improvements in their performances cannot be realistically expected. Typically the feeds of the reflectors are realized with high performance corrugated horns [2] that can be optimized for high efficiency, high gain, low cross polarization, high or low focal to diameter (F/D) ratio etc...

Typical feed horns can be lengthy and bulky, especially at low frequencies. This aspect alone constitutes a significant drive to find alternative technologies, more suited to mass production, that can be used to obtain equally performing feeds. In particular it is useful to explore the possibility of using printed circuit board technology to upgrade the performances of a moderately sized (in terms of the wavelength) waveguide horn, so that the resulting feed can be used to efficiently illuminate a reflector antenna.

Recent investigations seem to indicate that Electromagnetic Band Gap (EBG) super-layers could be used to obtain this goal. In [3], [4], [5] it was indicated that EBG super-strates could be adopted to increase the directivity of an aperture that is small in terms of the wavelength. However the directivity alone is not sufficient to qualify a feed pattern as good to centrally feed a reflector antenna, especially for medium or small $\mathrm{F} / \mathrm{D}$ ratios. In fact the global aperture efficiency results from the balance between spill over $\left(\eta_{s p}\right)$ and taper efficiency $\left(\eta_{\text {tap }}\right)$. It is well known that reducing the spill with a highly directive feed that creates a low edge taper will result in a poor taper efficiency. Thus, in this paper, the use of EBG dielectric super-layers is proposed to upgrade the small aperture pattern in a more sophisticated way: the emphasis will be on pattern shaping rather than on directivity increase alone.

An angular filtering effect that maximizes the reflector efficiency can be obtained: in this paper we will aim at the theoretical $\sec ^{4}(\theta / 2)$, [6] (see Fig. 1) that relates to center fed reflectors. In this article the term aperture ef-

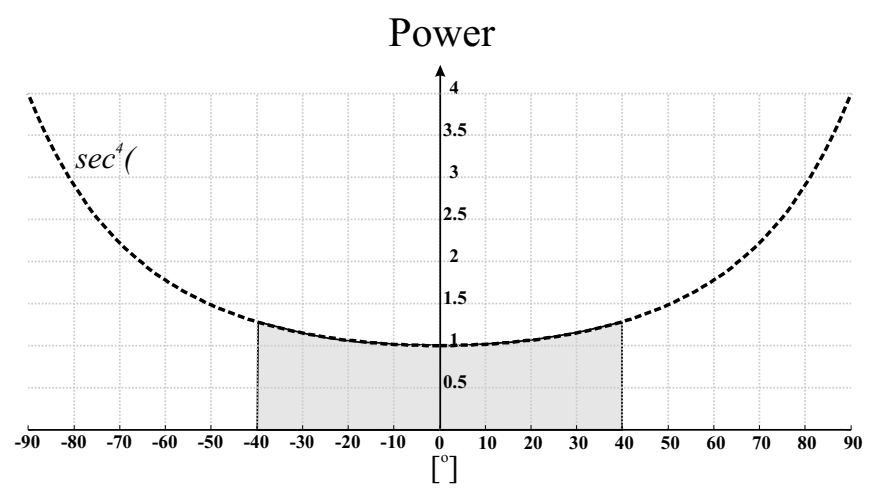

Figure 1. Ideal feed radiation pattern for maximum efficiency center fed reflectors depending on the reflector rim $\left(\theta_{0}\right)$.

ficiency is used to indicate the product of the spill over efficiency $\eta_{s p}$, the taper efficiency $\eta_{t a p}$ and the phase efficiency $\eta_{p h}: \eta_{a p}=\eta_{s p} \eta_{t a p} \eta_{p h}$. For the definitions in this paper we refer to [6]. These parameters are mostly associated to the relation between the feed pattern and the Focal distance to Diameter (F/D) ratio of the reflector.

Since we will consider center fed reflector another important factor that should be accounted for in a realistic design is the blockage efficiency $\eta_{b l}$. However, the blockage efficiency largely depends on the actual diameter, D, of the reflector. This is not taken as a design parameter in this article. For this reason the blockage will not be accounted for and the readers interested in actual efficiency figures will be required to scale the theoretical figures given here to account for actual blockage in systems of their interest.

\section{FEED DESIGN FOR HIGH EFFICIENCY RE- FLECTOR}

The geometry of the structure under investigation is described in Fig.2. It consists of a square metallic waveg- 
uide opening in a infinitely extended ground plane covered by a period arrangement of infinite dielectric layers. Note that the effects of the finiteness of the ground plane and of the dielectric slabs will be accounted for in the final analysis stage rather than in the following preliminary design phase. The reference system has its $\mathrm{z}$ axis normal to the ground plane and to the dielectric stratifications, the geometrical parameters are specified in the inset. For simplicity, in this paper we will consider only the two orthogonal $T E_{10}$ modes. This approximation will appear to be valid taking into account the long tapering of the feeding waveguide eventually implemented.

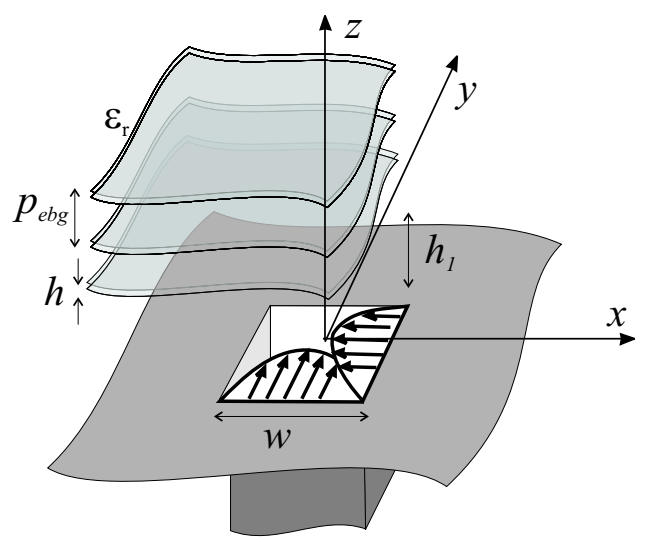

Figure 2. Square metallic waveguide of dimension $w$ in a infinitely extended ground plane covered by a period arrangement of infinite dielectric layers. The dielectric layers have a thickness $h$ and dielectric constant $\epsilon_{r}$, while period between them is $p_{\text {ebg. }}$. The distance between the ground plane and the first dielectric layer is $h_{1}$.

In this section we will present a design that could be used to enhance the properties of a center fed reflector characterized by optimum $F / D=0.72$. The base is a waveguide aperture to about $l=1.08 \lambda_{0}$. The simulations shown in this section are performed using the code described in [7].

The aim of the design is to obtain an impedance matching for the feeding waveguide. It is possible to fine tune the distance between the ground plane and the dielectric layers $h_{1}$ so that the external admittance, $Y^{e b g}$, would be real. The calculated reflection coefficient for the EBG covered feed is shown in Fig.3 as a function of the normalized frequency. The case of the waveguide in free space is shown for reference purposes.

The optimization of the matching has been performed simultaneously with the optimization of the radiation patterns: that is reducing the distance $p_{e b g}$ to compensate for the shift of the dominant pole location caused by the increase of $h_{1}$. The patterns are shown in Fig.4 at the central frequency. The phase distribution at the reflector aperture is shown in Fig.5. It is instructive to observe that the maximum variation of the phase is centered around the pointing angle of the dominant leaky wave (in the present example this is $32^{\circ}$ ). This phase variation

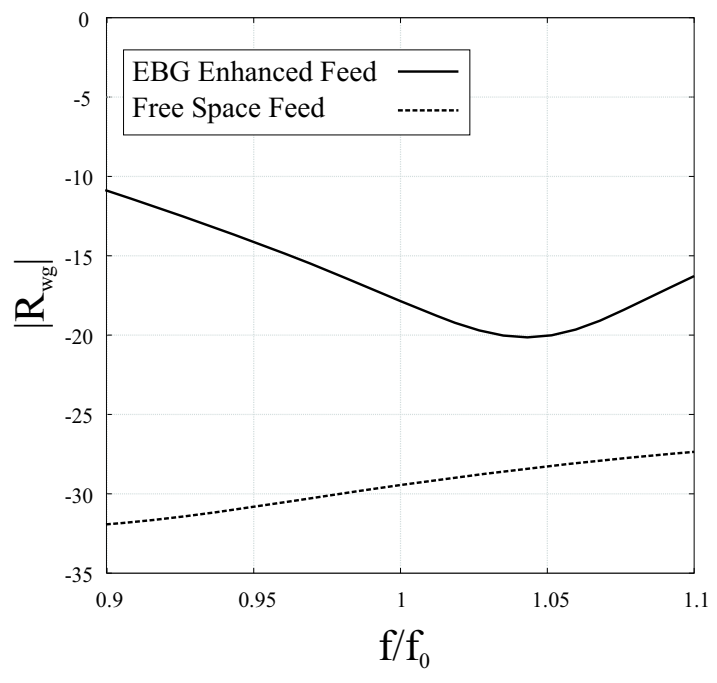

Figure 3. Reflection coefficient of the final design as a function of the normalized frequency $\left(\epsilon_{r}=2.2, h_{1}=14.5\right.$ $\left.\mathrm{mm}, p_{\text {ebg }}=13.8 \mathrm{~mm}, \mathrm{~h}=4.75 \mathrm{~mm}, f_{0}=10.64 \mathrm{GHz}\right)$. The reflection coefficient of the waveguide in free space is also shown.

property is a cause of reduction of the aperture efficiency. However, for the present design based on low dielectric contrasts it is not a problem. Overall the radiation pattern is well behaved in amplitude and in phase. To get a first estimation of the quality of this feed its radiation patterns have been used to feed a circularly symmetric reflector. The efficiencies of the ensemble feed + reflector have been calculated using an in house developed code based on standard PO approximation for the reflector radiation integral.

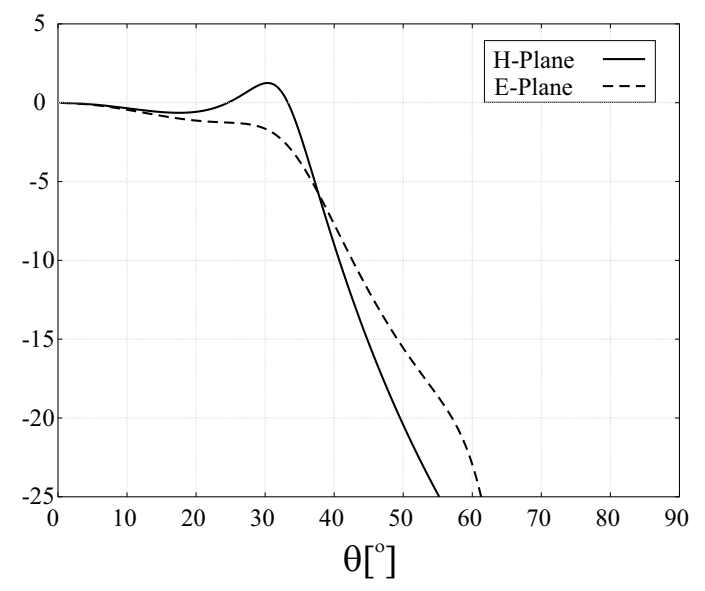

Figure 4. E and H-plane radiation patterns of the optimized structure of Fig.3 at the central frequency.

This feed reflector ensemble reaches an aperture efficiency of $\eta_{a p}=87.5 \%$. As shown in Fig. 6, the curve marked infinite, the high efficiency is obtained over a 


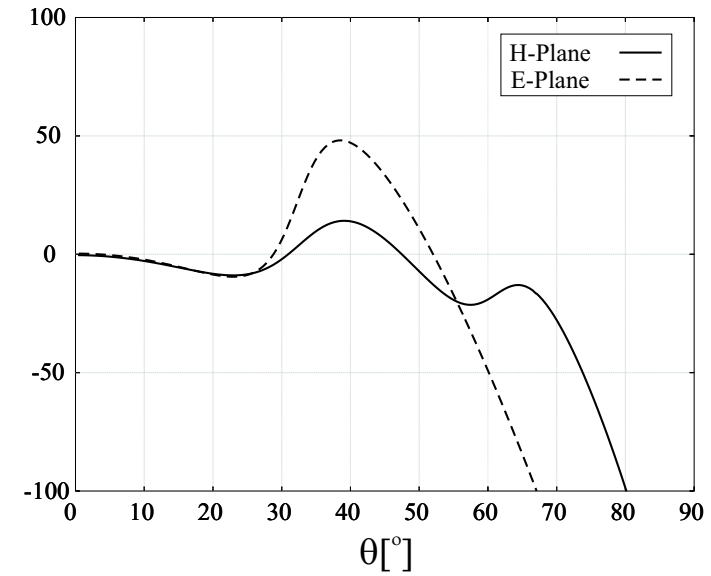

Figure 5. E and H-plane phase distribution at the reflector aperture of the optimized structure of Fig. 3 at the central frequency.

relatively large $\mathrm{BW}$ since $\eta_{a p}>84 \%$ is maintained over a $10 \%$ BW.

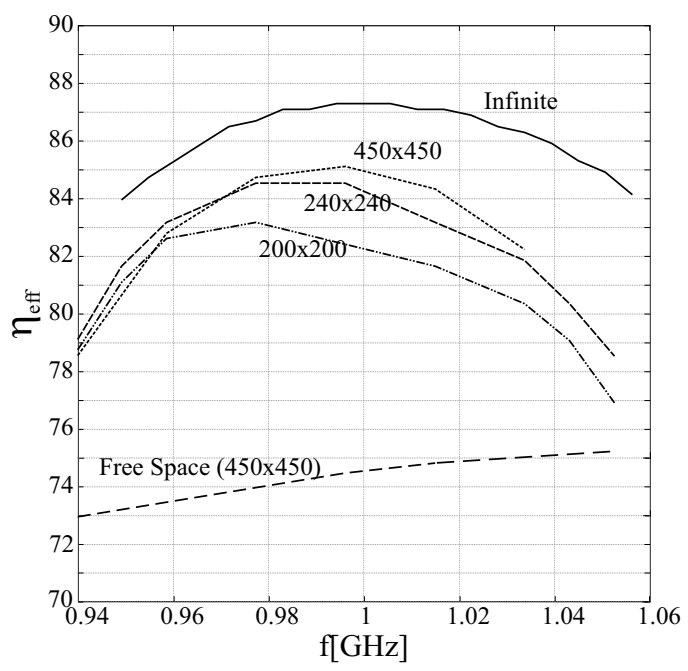

Figure 6. Aperture Efficiency as a function of the normalized frequency.

In the same figure are also reported the efficiencies as predicted using the fields obtained with the commercial code MSW CST [8]. With this code it was possible to consider dielectric slabs and ground planes of a finite dimension. A parametric analysis has been performed and the final predicted efficiencies for slabs of dimensions $200 \mathrm{~mm}$ x $200 \mathrm{~mm}, 240 \mathrm{~mm} \times 240 \mathrm{~mm}$, and $450 \mathrm{~mm} \times 450 \mathrm{~mm}$ are consequently reported. The efficiencies obtained with CST are always smaller than the ones predicted assuming infinitely extended dielectric slabs.

\section{PROTOTYPE DEMONSTRATOR}

In order to validate the concept described in this paper a prototype of the EBG enhanced feed has been manufactured, Fig.7. A standard WR90 waveguide

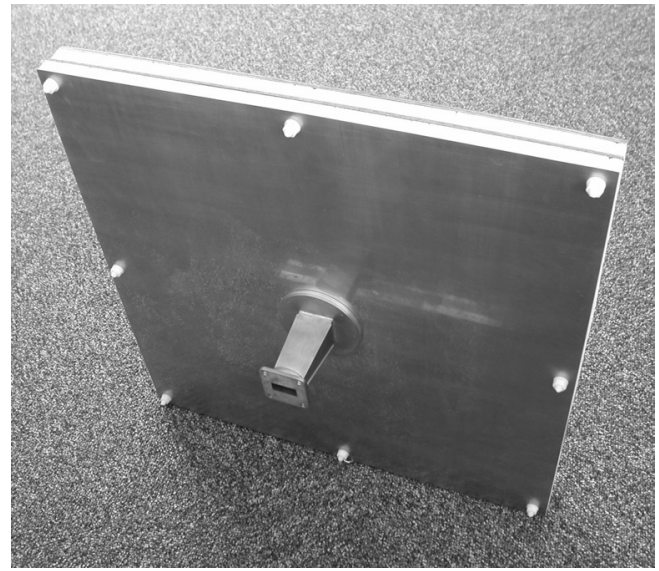

Figure 7. Photo of the prototype.

$(22.86 \mathrm{~mm} \times 10.16 \mathrm{~mm})$ is connected to the radiating aperture $(30.7 \mathrm{~mm} \times 30.7 \mathrm{~mm})$ via a section that was linearly flared in both directions. The largest slab case ( $425 \mathrm{~mm} \times 425 \mathrm{~mm}$ equivalent to $15 \lambda_{0} \times 15 \lambda_{0}$ ) was chosen for the prototype, since the aperture efficiency was expected to be the largest (see Fig.6). The frequency of operation has been chosen to be in the X-band, centered around $10.64 \mathrm{GHz}$ and to span over a $10 \%$ BW. Commercially available dielectric slabs with $\epsilon_{r}=2.2$, were separated one from the other and from the ground plane by means of two further foam slabs. The foam was initially thought of as having dielectric constant equal to the one of free space. The reflection coefficient as a function of the frequency has been measured and is shown in Fig. 8. Both the real and the normalized frequency scales are shown in the abscissa (below and above the graph). The two scales highlight that the bandwidth where the realized antenna presents lower reflection coefficient is shifted toward lower frequencies by about $10 \%$ (covering the $9-10 \mathrm{GHz}$ band rather than the $10-11 \mathrm{GHz}$ band). This significant shift seems to be mainly associated with the following manufacturing imperfections:

- The foam dielectric constant has been measured to be 1.07 rather than 1 . This causes a $7 \%$ frequency shift in the location of the leaky wave pole.

- The distance between the ground plane and the first EBG layer has been found to be $15 \mathrm{~mm}$ rather than the design value of $14.5 \mathrm{~mm}$.

The patterns from the prototype have been measured over a large frequency band showing that the frequency shift is not only in the reflection coefficient but also in the radiated fields. Fig. 9 shows the $\mathrm{E}$ and $\mathrm{H}$ plane radiation 


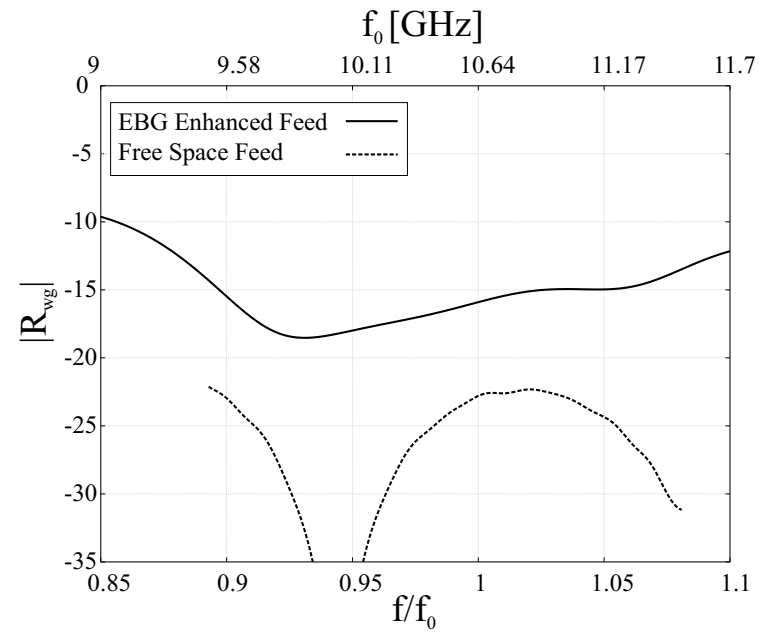

Figure 8. Measured reflection coefficient at the standard waveguide for the prototype as a function both of the frequency and of the normalized frequency.

patterns at the center of the bandwidth where the reflection coefficient is well matched $(9.6 \mathrm{GHz})$. Despite the shift in frequency, the patterns are very clean and resemble the designed one (Fig.4). One can observe the sharp drop off after $35^{\circ}$ as expected.

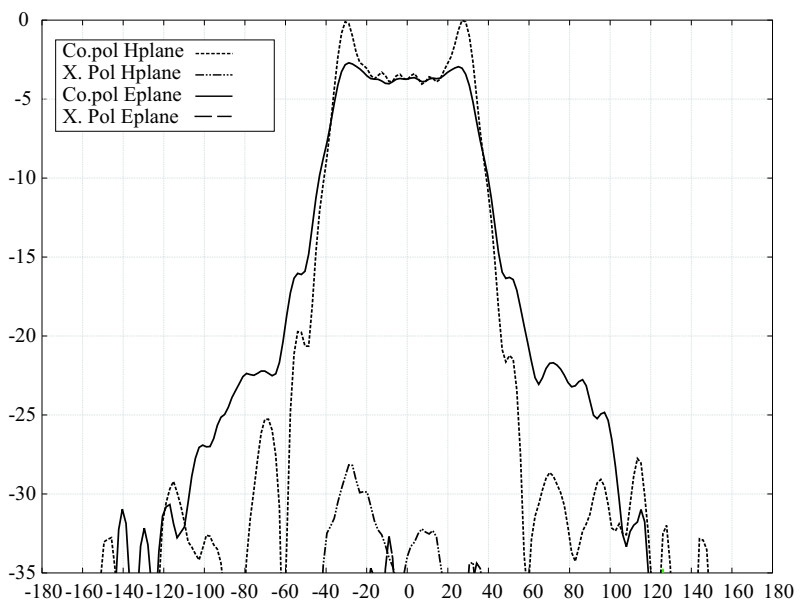

$\theta\left[{ }^{\circ}\right]$

Figure 9. Measured $E$ and $H$ plane radiation patterns at the central of operation of the prototype, $9.6 \mathrm{GHz}$.

From the measured feed patterns the efficiencies have been obtained for a center fed reflector of $F / D=0.72$ are shown in Fig.10. It is apparent that the bandwidth with aperture efficiency larger than $80 \%$ is shifted toward lower frequencies just like the reflection coefficient matching bandwidth. Despite the frequency shift the peak measured efficiency is only $2 \%$ lower than the peak predicted efficiency. Fig. 10 also shows the efficiencies associated to calculated feed patterns predicted from nu- merical simulations that include a value of $\epsilon_{r}=1.07$ for the dielectric constant of the slab. These simulations show reasonable resemblance with the measurements indicating, at least partially, the repeatability of the hardware.

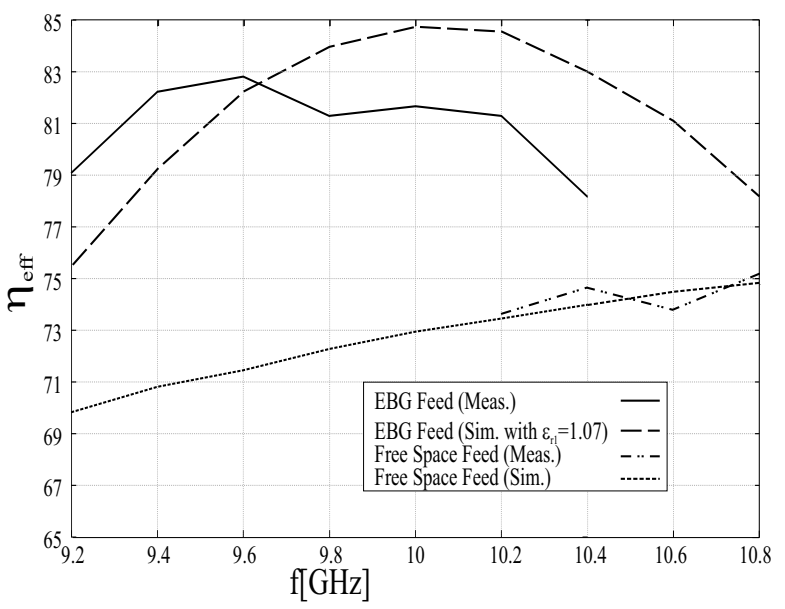

Figure 10. Measured aperture efficiency as a function of the frequency.

\section{CONCLUSIONS}

The aperture efficiency of reflector antennas fed by standard passive feeds is theoretically limited to $81 \%$. The radiation patterns from these feeds, e.g. horns, can approximately be modelled as $\cos ^{n}(\theta)$, where $n$ depends on the feed type and aperture size. In this contribution a novel design strategy is suggested. The aperture efficiencies of the feed+reflector systems are enhanced by accurately shaping the horn patterns to the ideal $\sec ^{4}(\theta / 2)$, using EBG super layers. Theoretically the design presented here shows $87 \%$ peak aperture efficiency. Also to be noted is that if the feed was to be used in excitation of a cylindrical reflector rather than a spherical one, $90 \%$ efficiency could have been theoretically predicted. In order to provide hardware demonstration of the concept a prototype EBG based feed has been manufactured and measured. The generated patterns, introduced in an analysis tool that simulates a center fed reflector system, provide an aperture efficiency larger than $80 \%$ over a $10 \%$ relative BW. A frequency shift affects the measurements, with respect to the design, whose origin has been clearly established in manufacturing inaccuracies.

\section{REFERENCES}

1. Y. Rahmat-Samii; "Chapter 15 Reflector Antennas" "Antenna Handbook" from Y.T.LO and S.W. Lee, , Van Nostrand Reinhold Company, New York 1988. 
2. Cha, A.; Bathker, D.; "Preliminary announcement of an 85 percent efficient reflector antenna" IEEE Transactions on Antennas and Propagation, Volume 31, Issue 2, Mar 1983 Page(s):341 - 342.

3. C. Cheype, C. Serier, M. Thevenot, T. Monediere, A. reineix, B. Jecko, "An Electromagnetic Bandgap Resonator Antenna" IEEE Transactions on Antennas and Propagation, Vol.50, no.9, September 2002, pp. 12851290.

4. Y. J. Lee, J.Yeo, R. Mittra, W.S. Park, “Application of Electromagnetic Bandgap Superstrates with Controllable defects for a Class of patch Antennas as Spatial Angular Filters" IEEE Transactions on Antennas and Propagation, Vol.53, no.1 January 2005, pp. 224-235.

5. Guerin, N.; Enoch, S.; Tayeb, G.; Sabouroux, P.; Vincent, P.; Legay, H. "A Metallic FabryPerot Directive Antenna" IEEE Transactions on Antennas and Propagation, Volume 54,Issue 1, Jan. 2006 Page(s):220 224.

6. Balanis Antenna Theory: Analysis and Design, Wiley Interscience 2005.

7. A. Neto, R. Bolt, G. Gerini, D. Schmidt "Multimode Equivalent Network for the Analysis of a Radome Covered Finite Array of Open Ended Waveguides" IEEE/AP-S -URSI meeting in Columbus, Ohio, June 2003.

8. CST Microwave Studio, User Manual Version 5.0, CST GmbH, Darmstadt, Germany. 